\title{
Hysteresis Modeling and Synchronization of a Class of RC-OTA Hysteretic-Jounce-Chaotic Oscillators
}

\author{
Leonardo Acho \\ CoDAlab, Departament de Matemàtica Aplicada III, \\ Escola Universitària d'Enginyeria Tècnica Industrial de Barcelona, \\ Universitat Politècnica de Catalunya, Comte d'Urgell,187, 08036 Barcelona, Spain \\ *Corresponding Author:leonardo.acho@upc.edu
}

Copyright (C)2013 Horizon Research Publishing All rights reserved.

\begin{abstract}
A class of RC-OTA hysteretic-chaotic oscillators has been previously reported using electronics; therefore, hysteresis is realized by an electronic circuit. To obtain a mathematical model of this RC-OTA chaotic-electronic device, hysteresis modeling turns an important issue. Here, we develop a new mathematical hysteretic model proposing a new jounce-chaotic oscillator. Chaosity test is proved using Poincaré theory. After that, a synchronization scheme is granted to synchronize our new jounce-chaotic oscillator (the transmitter) to a dynamics second-order system (the receiver).
\end{abstract}

Keywords Chaos, Synchronization, RC-OTA oscillator

\section{Introduction}

During recent years, many chaotic oscillators have been proposed. Some of them are based on hysteresis feedback ([11], [9]). However, these oscillators have been designed from electronic point of view. Nevertheless, hysteresis modeling is an important issue in mechanical and structural systems ([7]). Some hysteresis models are developed invoking physical laws. Meanwhile, others are heuristic ones. Moreover, [8] reported chaotic behavior in structures with hysteresis, in which hysteresis is governed by the well-known Bouc-Wen model. However, this Bouc-Wen model is not appropriate for the class of RC-OTA chaotic oscillators because it has more parameters than needed. Following this line of engineering modeling, we propose a new dynamic-hysteretic model which is able to capture hysteresis behavior for a class of RC-OTA hysteretic-chaotic oscillators designed, for instance, in [9] (where a pioneer study is given in [2]).

In mechanics, a jerk function is the time derivative of acceleration which, actually, is a third-order dynamic system. According to [10], some forms of jerk functions present chaos. And the time derivative of a jerk function might be called jounce ([10]), which is a fourth-order dynamic system. Using our dynamic-hysteretic model, and inspired by the RC-OTA architecture, we propose a fourth-order chaotic system named jounce-chaotic oscillator, and whose chaosity test is realized thought Poincaré theory.

By the other hand, chaos synchronization has gained an important attention among scientist (see, for instance, [1], [4], and [13], among others). This because some fields of engineering issues, e.g. secure communications, use chaos synchronization ([13], [1], and [3]). So far, many of the synchronization systems developed are granted to achieve chaos synchronization between two identical chaotic systems. The case of two different systems seems not to be complete developed ([13]). We also present a synchronization scheme where our jounce-chaotic system is synchronized with a second order system.

The structure of the paper is as follows. Section two presents a review of the simple mathematical model of a class of RC-OTA hysteretic-chaotic oscillators. Our new dynamic-hysteretic model is presented in Section three together with numerical experiments. Our new jouncechaotic system is commented too. In Section four, our synchronization design is granted. Finally, Section five presents the conclusions.

\section{RC-OTA hysteretic chaotic systems}

According to [9], a dimensionless dynamic model of a class of RC-OTA hysteretic-chaotic oscillator is given by:

$$
\ddot{x}-2 \delta \dot{x}+x=p h(x)
$$


where $\delta$ and $p$ are the system parameters. The hysteresis function $h(x)$ is shown in Fig. 1. This system presents chaos with $\delta=0.05$, and $p=1([9])$.

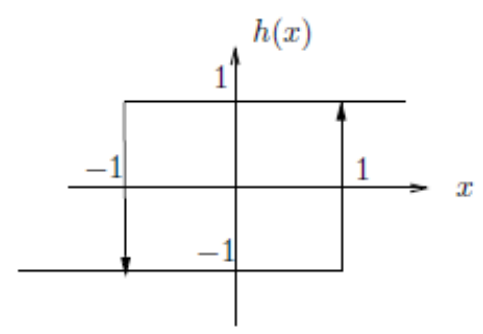

Figure 1. Normalized hysteretic function.

\section{Hysteresis modeling and jounce-chaotic system}

a

Hysteresis behavior is recognized as a system with memory. One way to capture hysteresis is by using a dynamic system. For instance, the new hysteretic system:

$$
\dot{z}=\alpha(-z+\operatorname{bsgn}(x+\operatorname{asgn}(z))),
$$

can reproduce the hysteretic behavior shown in Fig. 2, where $a$ and $b$ are the hysteresis curve parameters. The speed transition between $b$ and $-b$ is governed by the positive parameter $\alpha ; \operatorname{sgn}(\cdot)$ is the signum function. For instance, if $a=b=1$ and $\alpha=10$, the system (2) is:

$$
\dot{z}=10(-z+\operatorname{sgn}(x+\operatorname{sgn}(z))) .
$$

Using $x=10 \sin (t)$ and $z(0)=0$, the simulation result of system (3) is shown in Fig. 3 .

Next, we program a chaotic oscillator equivalent to (1):

$$
\begin{array}{ll}
\ddot{x} & -0.1 \dot{x}+x=z \\
\dot{z} & =10(-z+\operatorname{sgn}(x+\operatorname{sgn}(z))) .
\end{array}
$$

Fig. 4 shows the simulation result. The obtained chaotic attractor is the same as that shown in $[9$, Fig. $6]$.

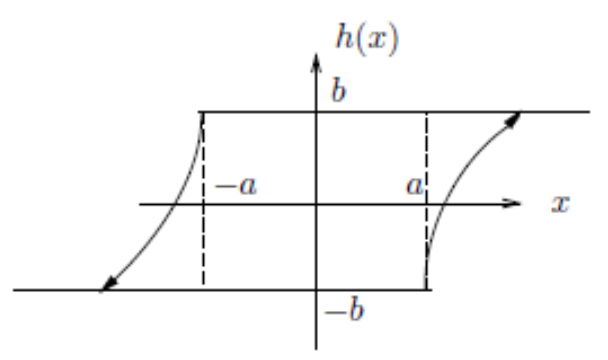

Figure 2. Hysteretic behavior.

To extend the previous system into a jounce-chaotic realization, consider the next propose:

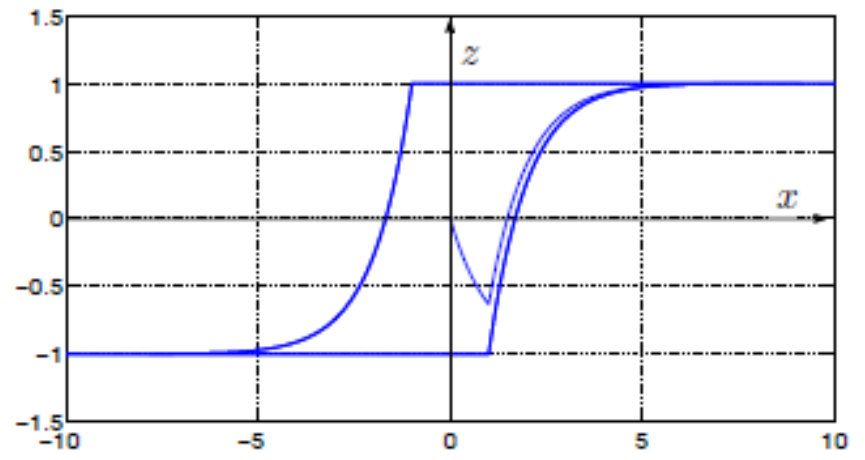

Figure 3. Simulation result.

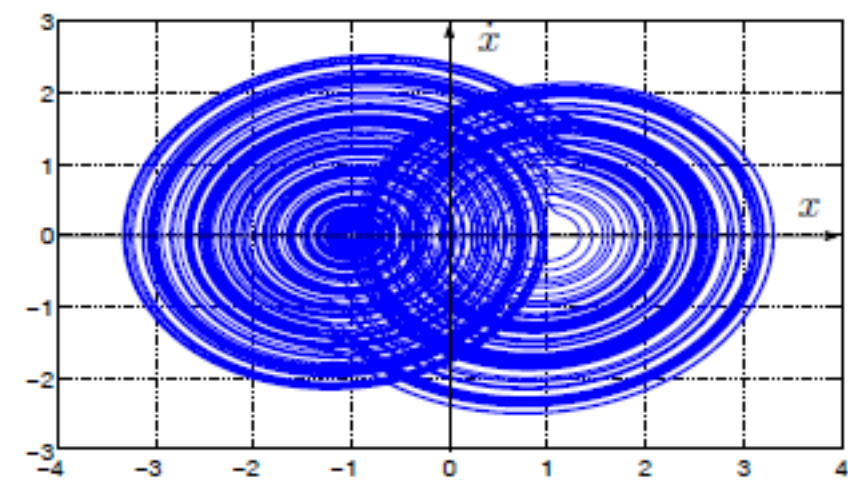

Figure 4. Chaotic attractor with initial conditions $x(0)=\dot{x}(0)=$ 0 and $z(0)=0.1$.

$$
\begin{aligned}
& \dot{x}_{1}=x_{2}, \\
& \dot{x}_{2}=z_{1}+z_{2}+0.1 x_{2}-x_{1}, \\
& \dot{z}_{1}=10\left(-z_{1}+\operatorname{sign}\left(x_{1}-1+\operatorname{sign}\left(z_{1}\right)\right)+0.5\right), \\
& \dot{z}_{2}=10\left(-z_{2}+\operatorname{sign}\left(x_{1}+1+\operatorname{sign}\left(z_{2}\right)\right)-0.5\right) .
\end{aligned}
$$

Figures 5 and 6 show the jounce-chaotic attractor of the system (4)-(7). Fig.7 shows the Poincaré map for the attractor shown in Fig. 6 using the Poincaré section a plane parallel to the plane $z_{1}-x_{2}$ and located at $x_{1}=1$. According to [12], this map consists of points filling up a region implying chaos. So, this Poncaré-map experiment is our chaosity test to our jounce-chaotic system. By the other hand, Fig. 8 displays $x_{1}(t)$ and $x_{2}(t)$ versus time. With a small change on the initial condition of $x_{1}$ from 0 to 0.001 (the remaining initial conditions were the same than the previous numerical experiment), the numerical results are displayed in Fig. 9. We thus have a dynamic system that presents bounded trajectory solutions that is highly sensitive to initial conditions and whose signals are random-like. These are the properties of a chaotic system.

\section{Synchronization}

Let us introduce the following system:

$$
\begin{aligned}
& \dot{y}_{1}=y_{2}, \\
& \dot{y}_{2}=z_{1}+z_{2}+0.2 x_{2}-y_{1}-0.1 y_{2}
\end{aligned}
$$




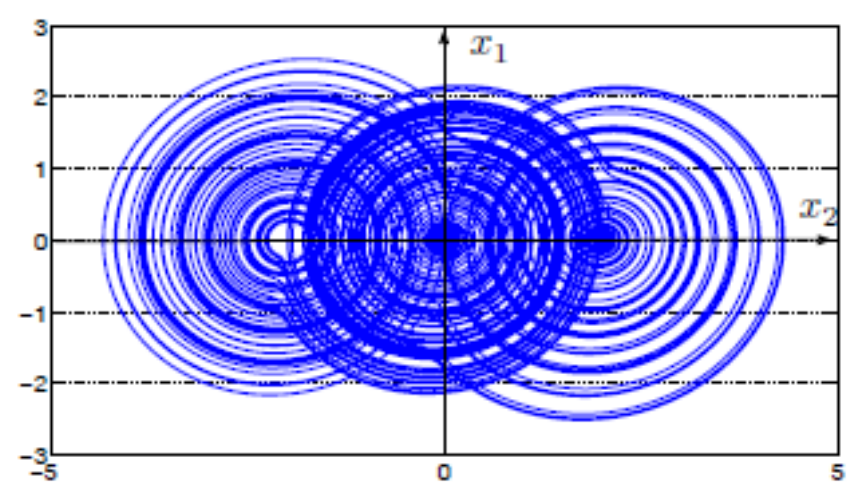

Figure 5. Jounce-chaotic attractor with initial conditions $x_{1}(0)=x_{2}(0)=z_{1}(0)=0$ and $z_{2}(0)=0.1$.

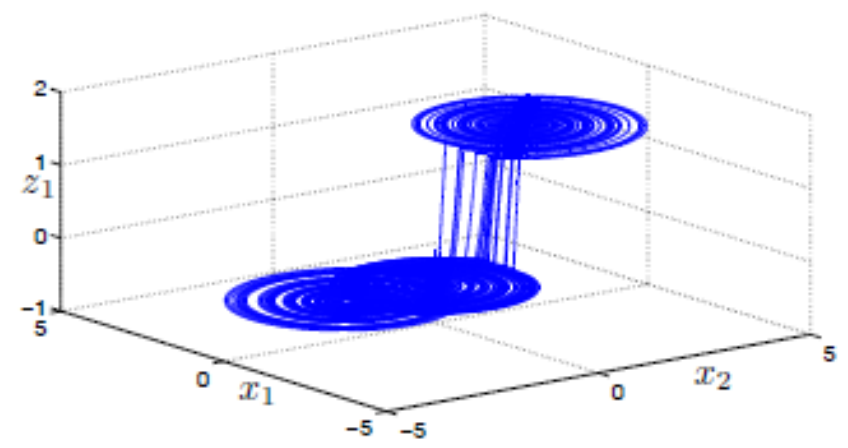

Figure 6. 3-D jounce-chaotic attractor.

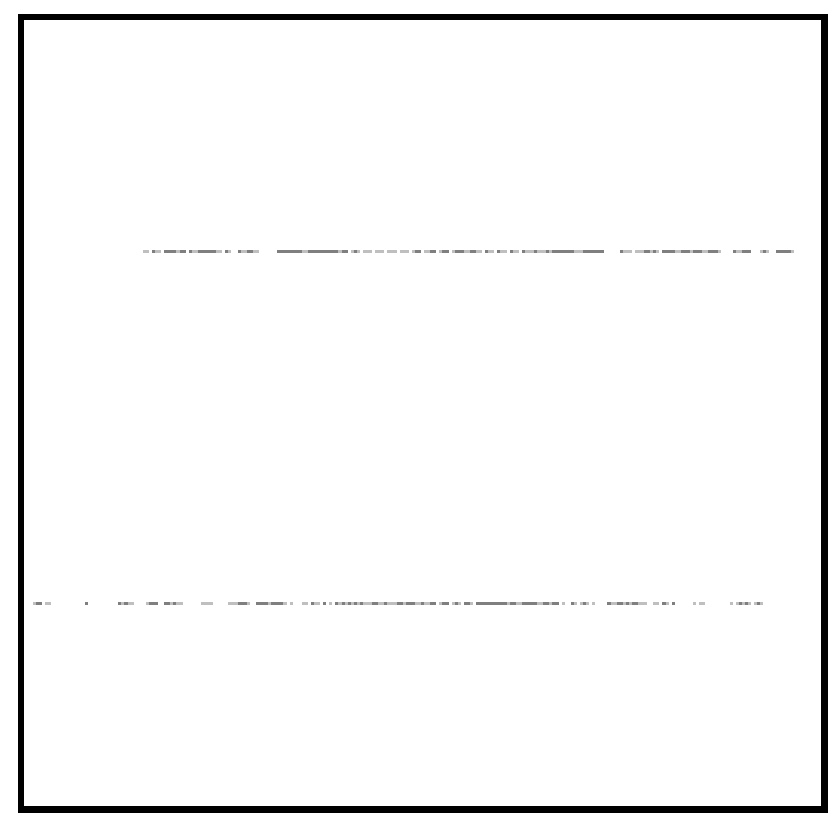

Figure 7. Poincaré Map.

where $z_{1}:=z_{1}(t), z_{2}:=z_{2}(t)$, and $x_{2}:=x_{2}(t)$ arrive from the jounce-chaotic system (4)-(7). At this point, the jounce-chaotic system (4)-(7) represents the transmitter and the system (8)-(9) the receiver. Fig 10 gives a schematic representation of our the synchronization design.

It is said that the receiver is synchronized with the transmitter if $y_{1}(t)$ converges to $x_{1}(t)$ and $y_{2}(t)$ converges to $x_{2}(t)$ as time goes on (and for any initial conditions
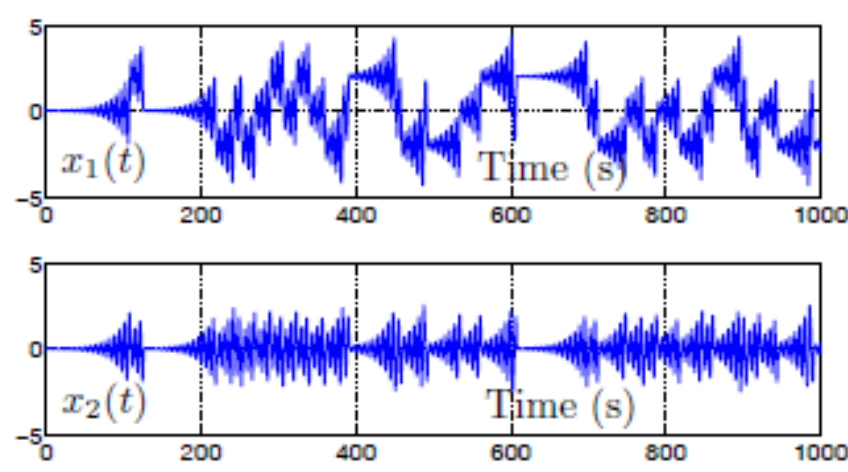

Figure 8. Simulation results: $x_{1}(t)$ and $x_{2}(t)$ versus time.

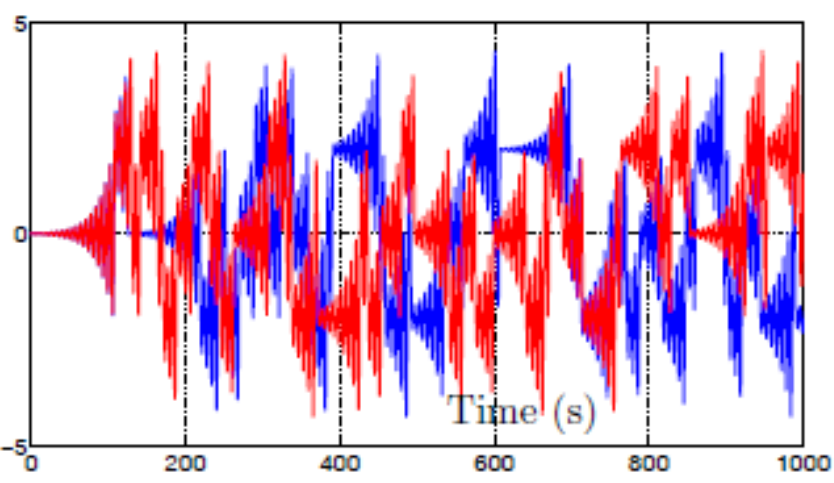

Figure 9. High sensitivity test on the initial conditions: blue line with $x_{1}(0)=0$ and red line with $x_{1}(0)=0.001$.

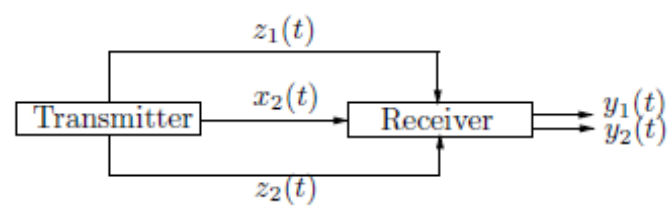

Figure 10. Synchronization block diagram.

$y_{1}(0), x_{1}(0), y_{2}(0)$, and $\left.x_{2}(0)\right)^{1}$. This fact can be proved as follows. Consider the signal errors given by

$$
\begin{aligned}
& e_{1}=x_{1}-y_{1}, \\
& e_{2}=x_{2}-y_{2} .
\end{aligned}
$$

Then, after some basic manipulations, we obtain:

$$
\begin{aligned}
& \dot{e}_{1}=e_{2}, \\
& \dot{e}_{2}=-e_{1}-0.1 e_{2} .
\end{aligned}
$$

The above system represents an exponential stable dynamics. Simulation results are shown in Fig. 11.

Remark 1 On synchronization of chaotic systems, it is used to test the synchronization performance by adding a noisy signal to the lines of the channel communication. This noisy signal is a kind of common noise because it is induced simultaneously on each communication lines $([5])$. But, from the technological point of view, common noisy signals are easily to remove via Instrumentation Amplifiers. For instance, according to ([5], page 85), the common noise induced

\footnotetext{
${ }^{1}$ Note that signals $z_{1}(t)$ and $z_{2}(t)$ are bounded for all $t \geq 0$.
} 

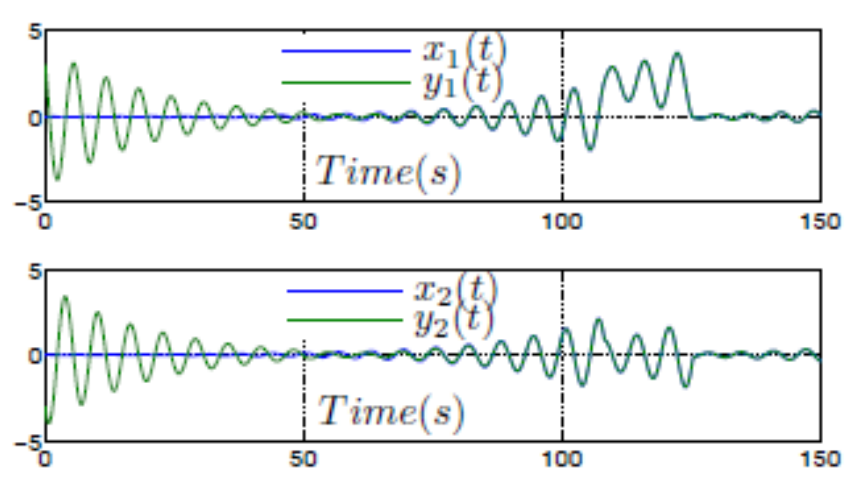

Figure 11. Simulation results with initial conditions $x_{1}(0)=$ $x_{2}(0)=z_{1}(0)=0, z_{2}(0)=0.1, y_{1}(0)=3$ and $y_{2}(0)=-3$.

on the communication lines can be reduced $140 d B$; i.e., it can be attenuate by a factor of $10^{14}$. By the other hand, employing fiber optics, noisy signals are practically unnoticed on our communication system.

Remark 2 The communication scheme shown in Fig. 10 requires three lines of communication. However, it is possible to implement a multiplexing communication technique to drive a single line of communication (Website http://en.wikipedia.org/wiki/Multiplexing).

\section{Conclusions}

A new hysteretic-jounce-chaotic system has been designed along with a synchronization scheme. According to numerical experiments, chaos synchronization between two different systems can be achieved. This fact was theoretically proved too.

\section{Acknowledgements}

This work was supported by grant number DPI201232375/FEDER from the Spanish Ministry of Economics and Competitiveness.

\section{REFERENCES}

[1] L. Acho, Expanded Lorenz systems and chaotic secure communication systems design,J. of Circuits, Systems, and Computers, Vol. 15, No. 4, 607-614, 2006.

[2] L. Acho, and Y. Vidal, Hysteresis modeling of a class of RC-OTA hysteretic-chaotic generators, 4th International Conference on Physics and Control (PhysCon), León, Spain, 2011.

[3] T.-L. Carroll, and L. M. Pecora, Synchronization chaotic circuits, IEEE Trans. on Circ. Syst., Vol. 38, 453-456, 1991.

[4] G. Chen, and X. Dong, From chaos to order: methodologies, perspectives and applications, World Scientific Pu., Singapore, 1998.

[5] S. Franco, Design with Operational Amplifiers and Analog Integrated Circuits, McGraw-Hill, N.Y., USA, Third Edition, 2002.

[6] H. Fengling, L. Jinhu, Y. C. Xinghuo, and C. Guanrong, Dynamical behaviours of a 3D hysteresis-based system, Chaos, Solitons and Fractals, Vol. 28, 182-192, 2006.

[7] M. Ismail, F. Ikhouane, and J. Rodellar, The hysteresis Bouc-Wen model, a Survey, Arch. Comput. Methods Eng., Vol. 16, 161-188, 2009.

[8] H. G. Li, and G. Meng, Nonlinear dynamics of a SDOF oscillator with BoucWen hysteresis, Chaos, Solitons and Fractals, Vol. 34, 337-343, 2007.

[9] S. Nakagawa, and T. Saito, An RC-OTA hysteresis chaos generator, IEEE Trans. on Circuits and Systems1: Fundamental Theory and Applications, Vol. 43, No. 12, 1019-1021, 1996.

[10] J. C. Sprott, Some simple chaotic jerk functions, Am. J. Phys., Vol. 65, No. 537, 537-543, 1997.

[11] M. Storace, and M- Parodi. Simple realisation of hysteresis chaos generator, Electronics Letters, Vol. 34, No. 1, 10-11, 1998.

[12] J. J.Thomsen, Vibrations and Stability: Advanced theory, analysis, and tools, Springer, Berlin, Germany, Second Edition, 2003.

[13] H. T. Yau, and J. J. Yan, Chaos synchronization of different chaotic systems sibjected to input nonlinearity, Applied Mathematics and Computation, Vol. 197, 775$788,2008$. 